in vivo $33: 1039-1049$ (2019)

doi:10.21873/invivo.11572

Review

\title{
Screening and Risk Assessment of Coronary Artery Disease in Patients With Type 2 Diabetes: An Updated Review
}

\author{
ALEXANDROS PATSOURAS ${ }^{1 *}$, PARASKEVI FARMAKI ${ }^{2}$, ANNA GARMPI $^{3}$, CHRISTOS DAMASKOS $^{4}$, \\ NIKOLAOS GARMPIS ${ }^{4}$, DIMITRIOS MANTAS ${ }^{4}$ and EVANGELOS DIAMANTIS ${ }^{5}$ \\ ${ }^{1}$ Medical School, National and Kapodistrian University of Athens, Athens, Greece; \\ ${ }^{2}$ First Department of Pediatrics, Agia Sofia Children's Hospital, National and \\ Kapodistrian University of Athens, Athens, Greece; \\ ${ }^{3}$ Internal Medicine Department, Laiko General Hospital, Medical School, National and \\ Kapodistrian University of Athens, Athens, Greece; \\ ${ }^{4}$ Second Department of Propedeutic Surgery, Laiko General Hospital, Medical School, \\ National and Kapodistrian University of Athens, Athens, Greece; \\ ${ }^{5}$ Health Center Peristeriou, Athens, Greece
}

\begin{abstract}
Current literature indicates that there is a strong correlation between coronary artery disease $(C A D)$ and type 2 diabetes. The arteriosclerotic progression occurs earlier and in a greater extent in the diabetic than in the non-diabetic population. In diabetic subjects, the detection of arterial disease does not always precede the development of an acute arterial incident. Herein, we reviewed studies published within the last 5 years in order to reveal the risk factors for coronary artery disease in patients with type 2 diabetes. In addition, we aimed to discuss how to diagnose in an early stage or even screen the presence of coronary artery disease in asymptomatic diabetic patients. Possible blood markers as predictors of CAD, which are mostly related to the lipidemic profile of subjects, are included in this review. Less invasive imaging methods than conventional coronary angiography, included in the article, are gradually used more in the diagnosis of CAD and show high effectiveness. Data from 23 articles with 22,350 patients having type 2 diabetes were summarized and presented descriptively.
\end{abstract}

This article is freely accessible online.

*These Authors contributed equally to this study.

Correspondence to: Dr. Christos Damaskos, MD, M.Sc., Ph.D., Second Department of Propedeutic Surgery, Laiko General Hospital, Medical School, National and Kapodistrian University of Athens, 17 Agiou Thoma Street, 11527 Athens, Greece. Tel: +30 6948467790, e-mail: x_damaskos@yahoo.gr

Key Words: Coronary, artery, disease, diabetes, atherosclerosis, computed, tomography, angiography, review.
The rates of diabetes are increasing worldwide. The scientific community estimates that the number of people living with diabetes will rise dramatically the following years and will reach the number of 592 million by 2035 (1). Diabetes mellitus has a wide range of complications which includes both microvascular (renal, retinal, and neuropathic disease) and macrovascular complications [vascular disease and coronary artery disease (CAD)] (2). The main system affected by diabetes, causing death, is the cardiovascular one. As a result, patients suffering from diabetes are prone to more severe cardiovascular diseases and have greater complication rates than non-diabetic patients (3).

Inflammatory elements, vascular smooth muscle cell proliferation and endothelial dysfunction, which characterize atherosclerosis, result in atherosclerotic plaque instability and progression (4-10). Atherosclerosis leading to CAD results in restriction of blood flow to the heart (11). It is common knowledge that the degree of stenosis varies among patients. Therefore, the clinical presentation of patients also varies from asymptomatic to stable angina and acute coronary syndrome (ACS), which includes unstable angina, stemi and non-stemi myocardial infraction (12).

Diabetes is regarded as a CAD risk equivalent. This means that diabetic patients are at risk of having coronary events alike non-diabetic patients, who previously had one (13). Many factors contribute to the appearance of CAD in diabetes type 2 patients and only $25 \%$ of these are already known (14). As CAD constitutes a challenging task among practitioners, the aim of our review is to present the correlation between type 2 diabetes mellitus and CAD, according to current scientific reports, and to reveal possible 
predictive factors that could be used as a screening and risk assessment tool for CAD in the future.

\section{Literature Review}

A search was conducted in MEDLINE (via PubMed) in order to retrieve articles from the period of time between 20142017. The search strategy was based on the use of keywords such as coronary artery disease, type 2 diabetes, coronary computed tomography, angiography and atherosclerosis. The PRISMA approach was used for the selection of the articles included in the review. A total of 41 records were identified. Following removal of the duplicates 28 records remained. These were screened and five were excluded mainly because they were only abstracts. The full-text articles assessed for eligibility were 23 and none of them was excluded. The inclusion process is demonstrated in Figure 1.

\section{Blood Factors as Predictors for CAD}

There is a variety of factors determining the risk of CAD, including blood markers, a common and useful tool for the prediction of CAD. In 2014, Huang et al., reported a study in which they compared sdLDL cholesterol (sdLDL C) levels between healthy individuals and diabetics with CAD in a group of Taiwanese people (Table I). It is known that, sdLDL particles are more atherogenic due to their characteristics, which include lower binding affinity to LDL receptors, higher penetration into subendothelial layer, longer half-life and lower resistance to oxidative stress. According to this study, sdLDL C combined with LDL C leads to better prediction of CAD in diabetic patients than LDL $\mathrm{C}$ alone. It is noteworthy that there was an age and sex effect on sdLDL-C as older people and males showed greater levels of sdLDL-C $(p<0.001)(15)$.

One year later, a study of 90 participants evaluated the possible association between atherosclerosis and glycated apo B and the later with the glycemic index and sdLDL (Table I) (16). It was shown that hyperglycemia and sdLDL are independently linked to glycation of apo B. They have suggested that the procedure of glycation and its results in the structure of vessels predisposes to atherosclerosis (16).

In addition, another study aimed to link young onset CAD to lipoprotein(a) $\{\mathrm{Lp}(\mathrm{a})\}$ in type 2 diabetic patients (Table I) (17). Based on previous studies, there was the hypothesis that $\mathrm{Lp}(\mathrm{a})$-associated cholesterol can promote coagulation, inflammation, carry proinflammatory oxidized phospholipids and prevent fibrinolysis (18). According to Chen's study there is significant evidence that higher $\mathrm{Lp}$ (a) levels are an independent factor predicting CAD patients with type 2 diabetes, suggesting that $\mathrm{Lp}$ (a) level measurement can be beneficial for type 2 diabetic patients in clinical practice (17).
Ozturk et al. have published a study of 158 subjects evaluating the correlation between CAD and blood galectin3 (Table I) (19). Galectin-3 is defined as a carbohydratebinding protein with anti-inflammatory and proinflammatory actions, depending on the inflammatory environment and the target cell or tissue. It has also been shown that high galectin-3 levels are positively associated with coronary atherosclerosis (both the extent and the type of plaque), High-sensitivity-C-Reactive Protein (hs-CRP) levels and BMI in diabetic patients (20).

Still in 2015, another study of 76 participants who were angiographically tested for the detection of CAD has estimated the role of insulin resistance and other markers of type 2 diabetes in the development of CAD (Table I) (21). This study has shown that low levels of insulin resistance and microalbuminuria, and female sex constitute negative factors for the development of CAD in patients suffering from diabetes type 2 for more than 10 years. Srinivasan's study suggested that these factors improve prognosis concerning the CAD profile (21).

A possible relationship between copeptin and cardiovascular mortality has also been tested (Table I) (22). Copeptin, a stable fragment of the vasopressin hormone, has been demonstrated to be an independent predictor for the appearance of diabetes mellitus (23). Several studies have demonstrated that in elderly population $\mathrm{CAD}$ and cardiovascular mortality rates increase as the level of blood copentin increases irrespective of diabetes mellitus. As a result, copeptin may be a useful tool for practitioners to assess cardiovascular risk stratification of primary prevention actions (23-25).

In 2016, Chubb et al., presented a study of 1,283 subjects aiming to examine the correlation between the concentration of $\mathrm{HCO}_{3}$ in blood and coronary heart disease CHD), heart failure and mortality (Table I). According to the study, there is an inverse association between serum bicarbonate and CHD events in type 2 diabetic patients (as serum bicarbonate levels decrease, the risk of CHD events increases). Additionally, heart failure and mortality were not demonstrated to be associated to serum bicarbonate levels (26).

In addition, the relationship between serum vaspin levels and prevalence of CAD has been investigated (Table I) (27). Vaspin is a factor having anti-inflammatory action. Reduced vaspin levels are related to increased CRP and visfatin levels (28). Vaspin, which is a ligand for VDAC/GRP78 complex in the surface of vascular cells, has a protective effect and prevents apoptosis of vascular cells via phosphatidylinositide 3 kinase-AKT (PI3K-AKT) signaling pathway (Figure 2) (29). Akt phosphorylates and inactivates BAD, which is a pro-apoptotic factor, inhibiting Bcl-xL (an antiapoptotic factor) (30). Hao's et al. study has indicated that diabetic patients with CAD have higher levels of serum vaspin compared to diabetics without $\mathrm{CAD}$ and healthy individuals (27). 


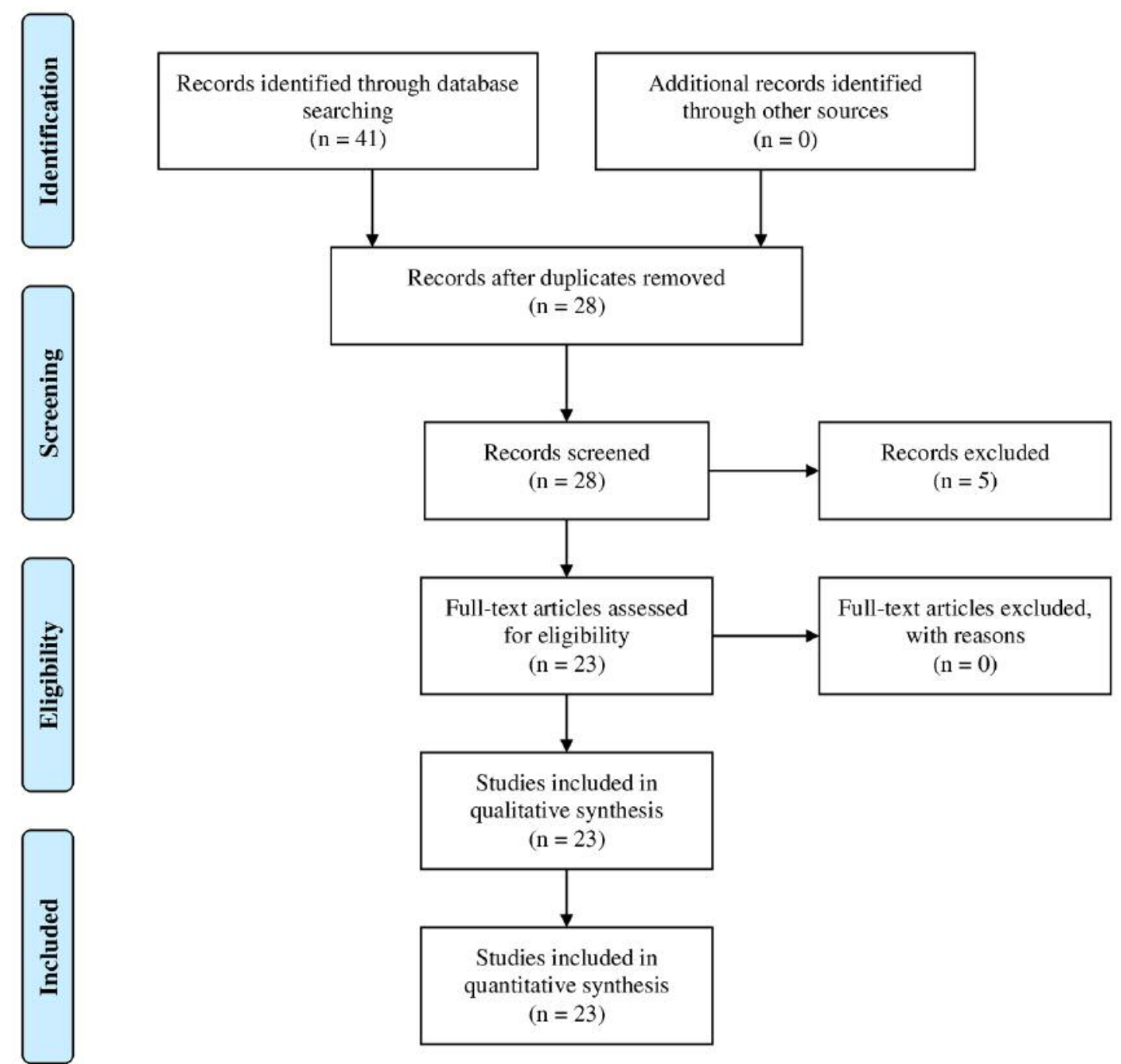

Figure 1. Prisma flow diagram for the current literature review.

Müller et al., have examined whether blood Gremlin-1 and macrophage migration inhibitory factor (MIF) might be related to $\mathrm{CAD}$ and the risk of acute coronary syndrome (ACS) (Table I) (31). MIF is a pro-inflammatory factor which regulates monocyte recruitment, leading to atheroprogression and plaque instability (32). Gremlin-1 is an antagonist of MIF preventing foam-cell formation induced by MIF and thus has an atheroprotective action (33). A study has shown that patients with type 2 diabetes and ACS had high levels of both Gremlin-1 and MIF. Additionally, the MIF/gremlin-1 ratio was high, as MIF increased more than gremlin-1. This ratio was demonstrated only in type 2 diabetes with ACS (31).

Valensi et al., have examined the association between asymptomatic myocardial ischemia and asymptomatic CAD with atherogenic dyslipidemia in a study that included 1080 subjects with type 2 diabetes (Table I). Atherogenic dyslipidemia is defined as low levels of high-density lipoprotein cholesterol and high levels of triglycerides. The clinical results confirmed the primary hypothesis, indicating an increased risk of atherogenic dyslipidemia and a possible molecular targeting of atherogenic dyslipidemia could offer improved clinical results (34).

\section{Imaging Methods as Predictors for CAD}

Imaging methods are in the forefront of the clinical diagnosis of CAD and the broad range of its complications. In 2014, Gyung-Min Park et al., examined a group of 557 asymptomatic participants who underwent coronary computed tomography angiography (CCTA) evaluation, as a screening method, and found that there is a positive 
in vivo $33: 1039-1049(2019)$

Table I. Clinical trials of blood markers as screening methods for coronary artery disease.

\begin{tabular}{|c|c|c|c|c|c|c|}
\hline Entry & Study & Design & Population & $\begin{array}{l}\text { Method of } \\
\text { screening }\end{array}$ & $\begin{array}{l}\text { Clinical } \\
\text { outcome }\end{array}$ & Conclusion \\
\hline 1 & $\begin{array}{l}\text { Huang } \\
\text { et al. }\end{array}$ & $\begin{array}{c}\text { Case control } \\
\text { study }\end{array}$ & $\begin{array}{l}453 \text { individuals } \\
\text { classified in three } \\
\text { groups, healthy, T2DM } \\
\text { with and without CAD }\end{array}$ & Blood sample & $\begin{array}{l}\text { CAD in association with } \\
\text { both SdLDL-C and LDL-C }\end{array}$ & $\begin{array}{l}\text { High levels of LDL-C and } \\
\text { SdLDL-C increase the risk } \\
\text { of CAD in T2DM }(\mathrm{OR}=4.97 \text {, } \\
95 \% \mathrm{CI}=1.96-12.57, p=0.001) \text {. } \\
\text { Measurement of both factors } \\
\text { offers better assessment } \\
\text { concerning the risk of T2DM } \\
\text { with CAD than LDL alone. }\end{array}$ \\
\hline 2 & $\begin{array}{l}\text { Dev } \\
\text { et al. }\end{array}$ & $\begin{array}{c}\text { Case control } \\
\text { study }\end{array}$ & $\begin{array}{l}45 \text { obese diabetic patients } \\
\text { between } 45 \text { to } 65 \text { years old } \\
\text { and } 45 \text { non-diabetic } \\
\text { obese individuals }\end{array}$ & Blood sample & $\begin{array}{l}\text { Relationship of subclinical } \\
\text { atherosclerosis } \\
\text { with Sd-LDL } \\
\text { and Apo B }\end{array}$ & $\begin{array}{l}\text { Serum LDL/apoB }<1.2 \text { is } \\
\text { considered atherogenic in } \\
\text { DM VS non-DM }(48.9 \% \\
v s .28 .9) \text {. There is significant } \\
\text { correlation between glycated } \\
\text { Apo B and postprandial } \\
\text { glucose }(p=0.001), \text { HbA1c } \\
(p=0.013) \text { and fasting } \\
\text { blood glucose }(p=0.000) .\end{array}$ \\
\hline 3 & $\begin{array}{l}\text { Chen } \\
\text { et al. }\end{array}$ & $\begin{array}{l}\text { Cross- } \\
\text { sectional } \\
\text { study }\end{array}$ & $\begin{array}{l}1254 \text { patients with young onset } \\
\text { CAD divided into DM } \\
\text { and non-DM }\end{array}$ & Blood sample & $\begin{array}{l}\text { Young onset CAD in } \\
\text { association with } \\
\text { serum } \mathrm{Lp}(\mathrm{a})\end{array}$ & $\begin{array}{l}\text { Lp(a) is an independent } \\
\text { predictor for high } \\
\text { Gensini score }(\mathrm{OR}=1.82 \\
95 \% \mathrm{CI}=1.10-3.12, p=0.029) \\
\text { and the severity of ypung } \\
\text { onset CAD in T2DM. }\end{array}$ \\
\hline 4 & $\begin{array}{l}\text { Ozturk } \\
\text { et al. }\end{array}$ & $\begin{array}{l}\text { Case control } \\
\text { study }\end{array}$ & $\begin{array}{l}158 \mathrm{~T} 2 \text { diabetic patients } \\
\text { divided into CAD } \\
\text { and non-CAD }\end{array}$ & Blood sample & $\begin{array}{l}\text { Evaluation of CAD in } \\
\text { correlation with } \\
\text { blood galectin-3 }\end{array}$ & $\begin{array}{l}\text { Galectin-3 was found to be a } \\
\text { significant independent } \\
\text { predictor of coronary } \\
\text { atherosclerosis in DM2 } \\
(\mathrm{OR}=1.003,95 \% \mathrm{CI}= \\
1002-1004, p<0.001) \text { and } \\
\text { CAD patients show higher } \\
\text { levels of serum galectin-3 } \\
\text { than non-CAD } \\
(1412.0 \pm 441.7 v s \text {. } \\
830.2 \pm 434.9 \mathrm{pg} / \mathrm{ml}, p<0.001) .\end{array}$ \\
\hline 5 & $\begin{array}{l}\text { Srinivasan } \\
\quad \text { et al. }\end{array}$ & $\begin{array}{l}\text { Case control } \\
\text { study }\end{array}$ & $\begin{array}{l}76 \text { patients with DM } \\
\text { for more than } 10 \text { years }\end{array}$ & $\begin{array}{c}\text { Coronary } \\
\text { angiogram }\end{array}$ & $\begin{array}{l}\text { Evaluation of CAD and } \\
\text { MACE at } 1 \text { year } \\
\text { (revascularization, } \\
\text { non-fatal MI, death) }\end{array}$ & $\begin{array}{c}\text { HOMA -IR }<2.5(\mathrm{OR}=9.09, \\
95 \% \mathrm{CI}=1.91-41.83, p=0.005), \\
\text { microalb }<20 \mathrm{mg} / 1(\mathrm{OR}=4.57, \\
95 \% \mathrm{CI}=1.17-17.85, p=0.029) \\
\text { and females }(\mathrm{OR}=7.91, \\
95 \% \mathrm{CI}=1.55-40.38, p=0.013) \\
\text { are the factors that appear to } \\
\text { be associated with NO CAD. } \\
\text { No MACE happened when } \\
\text { HOMA -IR }<2.5(p=0.001) .\end{array}$ \\
\hline 6 & $\begin{array}{l}\text { Tasevska } \\
\text { et al. }\end{array}$ & $\begin{array}{l}\text { Prospective } \\
\text { cohort } \\
\text { study }\end{array}$ & $\begin{array}{l}4873 \text { subjects of } 70 \text { years } \\
\text { old, approximately, } \\
\text { both diabetics and non- } \\
\text { diabetics without } \\
\text { history of known CAD }\end{array}$ & Blood sample & $\begin{array}{l}\text { All-cause mortality } \\
\text { including } \\
\text { cardiovascular and } \\
\text { CAD events (first MI } \\
\text { and revascularization) }\end{array}$ & $\begin{array}{l}\text { Significant correlation between } \\
\text { copeptin and cardiovascular } \\
\text { mortality }(\mathrm{HR}=1.36, \\
95 \% \mathrm{CI}=1.21-1.53, p<0.001) \\
\text { and CAD development } \\
(\mathrm{HR}=1.20,95 \% \mathrm{CI}=1.08-1.33, \\
p=0.001) \text { both in the presence } \\
\text { and the absence of DM. }\end{array}$ \\
\hline 7 & $\begin{array}{l}\text { Chubb } \\
\text { et al. }\end{array}$ & $\begin{array}{l}\text { Prospective } \\
\text { cohort } \\
\text { study }\end{array}$ & $1283 \mathrm{~T} 2 \mathrm{DM}$ patients & $\mathrm{HCO} 3$ & $\begin{array}{c}\text { Death or first } \\
\text { hospitalization } \\
\text { due to cardiac events } \\
\text { (HF, CHD) }\end{array}$ & $\begin{array}{c}\text { There is an inverse relationship } \\
\text { between } \mathrm{HCO}_{3} \text { and risk of CHD } \\
\text { events in } \mathrm{T}_{2} \mathrm{DM} \text {. Increase by } \\
1 \mathrm{mmol} / 1 \text { of } \mathrm{HCO}_{3} \text { reduces } \\
5 \% \text { the risk of } \mathrm{CHD} \\
\text { (HR=0.95, } 95 \% \mathrm{CI}=0.92-0.99) .\end{array}$ \\
\hline
\end{tabular}


Table I. Continued

\begin{tabular}{|c|c|c|c|c|c|c|}
\hline Entry & Study & Design & Population & $\begin{array}{l}\text { Method of } \\
\text { screening }\end{array}$ & $\begin{array}{l}\text { Clinical } \\
\text { outcome }\end{array}$ & Conclusion \\
\hline 8 & $\begin{array}{l}\text { Hao } \\
\text { et al. }\end{array}$ & $\begin{array}{l}\text { Retrospective } \\
\text { cross- } \\
\text { sectional } \\
\text { study }\end{array}$ & $\begin{array}{c}228 \text { patients with } \\
\text { T2DM, } 45 \text { to } 65 \text { years } \\
\text { old and } 120 \\
\text { healthy participants }\end{array}$ & Blood sample & $\begin{array}{l}\text { CAD development } \\
\text { in relationship } \\
\text { with Vaspin }\end{array}$ & $\begin{array}{l}\text { Vaspin correlates with CAD } \\
\text { in DM2 }(p=0.001) \text {. Serum } \\
\text { vaspin levels are higher in } \\
\text { DM than non-DM }(p<0.05) \text {. } \\
\text { Serum vaspin levels are higher } \\
\text { in patients with T2DM and } \\
\text { CAD than patients } \\
\text { without CAD }(p<0.05) .\end{array}$ \\
\hline 9 & $\begin{array}{l}\text { Muller } \\
\text { et al. }\end{array}$ & $\begin{array}{l}\text { Cohort cross- } \\
\text { sectional } \\
\text { study }\end{array}$ & $\begin{array}{l}286 \text { symptomatic } \\
\text { CAD patients divided } \\
\text { into DM and non-DM }\end{array}$ & Blood sample & $\begin{array}{l}\text { Development of CAD in } \\
\text { correlation with MIF and } \\
\text { Gremlin-1 in blood sample }\end{array}$ & $\begin{array}{l}\text { In DM, higher plasma levels } \\
\text { of gremlin- } 1 \text { were found } \\
(p=0.001) \text {. Diabetic patients } \\
\text { with SAP or ACS show } \\
\text { higher levels of Gremlin- } 1 \\
\text { than non-diabetics with } \\
\text { SAP ( } p=0.008 \text { and } p=0.011 \text {, } \\
\text { respectively). Diabetic } \\
\text { patients with ACS rather } \\
\text { than SAP show higher } \\
\text { levels of MIF }(p<0.001) .\end{array}$ \\
\hline 10 & $\begin{array}{l}\text { Valensi } \\
\text { et al. }\end{array}$ & $\begin{array}{l}\text { Retrospective } \\
\text { cohort } \\
\text { study }\end{array}$ & $\begin{array}{l}1080 \text { asymptomatic } \\
\text { T2DM patients with } \\
\mathrm{LDL}<3.35 \mathrm{mmol} / \mathrm{L} \text {. }\end{array}$ & $\begin{array}{l}\text { Stress myocardial } \\
\text { scintigraphy, } \\
\text { blood sample }\end{array}$ & $\begin{array}{l}\text { SMI and silent CAD in } \\
\text { association with LDL, } \\
\text { HDL and TGs level }\end{array}$ & $\begin{array}{c}\text { Atherogenic dyslipidemia } \\
\text { associated with } \mathrm{SMI} \\
(\mathrm{OR}=1.8,95 \% \mathrm{CI}=1.0-3.3 \\
p<0.05) \text { and with silent } \\
\mathrm{CAD}(\mathrm{OR}=4.0,95 \% \mathrm{CI}= \\
1.7-9.2, p<0.001)\end{array}$ \\
\hline
\end{tabular}

CAD: Coronary artery disease; LDL: low density lipoprotein; SdLDL: small dense low-density lipoprotein; T2DM: type 2 diabetes mellitus; MACE: major adverse cardiovascular event; MI: myocardial infraction; HF: heart failure; CHD: coronary heart disease; ACS: acute coronary syndrome; Apo B: apolipoprotein B.

correlation between significant CAD and cardiac events, as well as lower survival rates (Table II) (35). Additionally, approximately $1 / 3$ of asymptomatic type 2 diabetic patients were found to have significant CAD. Thus, this study depicted the potential value of CCTA in the identification of asymptomatic type 2 diabetes being at high risk of cardiovascular complications (35).

One year later, a group of 6434 individuals more than 20 years old who underwent CCTA was tested (Table II). Compared to non-diabetics, type 2 diabetic patients presented asymptomatic $\mathrm{CAD}$ at higher rates, concerning the low and intermediate risk CAD mostly. Remarkably, diabetic patients with intermediate risk of CAD suffered more cardiac events than the ones with low risk CAD (36).

In 2015, 626 type 2 diabetic patients, not suffering from known cardiovascular disease were examined (Table II). During the study, the coronary artery calcium (CAC) score was compared using multi-detector computed tomography (MDCT), with the cardio-ankle vascular index (CAVI). According to the results, a CAVI $\geq 9.0$ or a CAC score $\geq 100$ were positively correlated with cardiovascular events. Although
CAC score has a greater predictive value, CAVI seems also a useful method for the risk stratification of asymptomatic type 2 diabetic patients. Importantly, CAVI presents a range of advantages, such as being a low-cost procedure, is easy to be calculated and is not affected by blood pressure (37).

The same year, investigators tested the usefulness of CCTA in CAD detection in comparison to invasive coronary angiography (ICA) which is regarded as the gold standard method was investigated in 48 type 2 diabetic asymptomatic patients (Table II). The results showed that CCTA has equal sensitivity with ICA. However, CCTA may present many falsepositive results reducing its effectiveness as an assessment tool for asymptomatic CAD in type 2 diabetics (38).

In another study, 506 type 2 diabetic patients asymptomatic for CAD were examined via multi-detector computed tomography (MDCT) (Table II). The research group demonstrated that $82 \%$ of men and $72 \%$ of women were suffering from CAD. Alongside, they assessed other clinical predictors, depending on the gender. Men with high levels of $\mathrm{HbA1c}$, longer duration of diabetes, retinopathy, dyslipidemia and other cardiovascular problems, as well as women with 
in vivo $33: 1039-1049(2019)$

Table II. Clinical trials of imaging methods as screening methods for coronary artery disease.

\begin{tabular}{|c|c|c|c|c|c|c|}
\hline Entry & Study & Design & Population & $\begin{array}{c}\text { Method of } \\
\text { screening }\end{array}$ & $\begin{array}{l}\text { Clinical } \\
\text { outcome }\end{array}$ & Conclusion \\
\hline 1 & $\begin{array}{l}\text { Park } \\
\text { et al. }\end{array}$ & $\begin{array}{l}\text { Prospective } \\
\text { cohort } \\
\text { study }\end{array}$ & $\begin{array}{l}557 \text { asymptomatic } \\
\text { T2DM patients } \\
\text { between } 30 \text { to } \\
80 \text { years old }\end{array}$ & CCTA & $\begin{array}{l}\text { Cardiovascular events } \\
\text { including non-fatal MI, } \\
\text { revascularization, } \\
\text { ACS or death }\end{array}$ & $\begin{array}{l}\text { Significant CAD on CCTA } \\
\text { is associated with more } \\
\text { cardiac }(7.1 \% \text { vs. } 0.5 \%) \text { events } \\
\text { and lower } 3 \text { year's event } \\
\text { free survival rates }(p<0.001) \text {. }\end{array}$ \\
\hline 2 & $\begin{array}{l}\text { Park } \\
\text { et al. }\end{array}$ & $\begin{array}{l}\text { Retrospective } \\
\text { case control } \\
\text { study?? }\end{array}$ & $\begin{array}{l}6434 \text { individuals more } \\
\text { than } 20 \text { years old } \\
\text { divided into diabetic } \\
\text { and non-diabetic }\end{array}$ & CCTA & $\begin{array}{l}\text { All-cause mortality, } \\
\text { revascularization } \\
\text { non-fatal MI, ACS }\end{array}$ & $\begin{array}{l}\text { Patients with DM have higher } \\
\text { CAC score and more severe } \\
\text { CAD than those without. } \\
\text { Diabetic patients with } \\
\text { intermediate rather than } \\
\text { low risk suffer more cardiac } \\
\text { events. }(\mathrm{HR}=3.197,95 \% \mathrm{CI}= \\
1.171-8.730, p=0.023) .\end{array}$ \\
\hline 3 & $\begin{array}{l}\text { Chung } \\
\text { et al. }\end{array}$ & $\begin{array}{l}\text { Retrospective } \\
\text { case control } \\
\text { study }\end{array}$ & $\begin{array}{l}626 \text { T2DM patients } \\
\text { more than } 35 \text { years old } \\
\text { not suffering from } \\
\text { known cardio- } \\
\text { vascular disease }\end{array}$ & $\begin{array}{c}\text { MDCT ABI } \\
\text { and CAVI } \\
\text { measurement by } \\
\text { CAVI instrument }\end{array}$ & $\begin{array}{l}\text { Any cardiovascular } \\
\text { event including death, } \\
\text { acute coronary } \\
\text { syndrome, stroke, } \\
\text { coronary } \\
\text { revascularization }\end{array}$ & $\begin{array}{c}\text { CAC score } \geq 1000 \text { is correlated } \\
\text { with total cardiovascular events } \\
(\mathrm{OR}=3.90,95 \% \mathrm{CI}=3.90-29.02, \\
p<0.001) \text {. CAVI } \geq 9 \text { is correlated } \\
\text { with total cardiovascular events } \\
(\mathrm{OR}=1.18,95 \% \mathrm{CI}=1.00-1.38, \\
p=0.049) \text {. A CAC score } \geq 1000 \\
\text { offers better predictive value } \\
\text { for total cardiovascular events } \\
\text { than CAVI } \geq 9 .\end{array}$ \\
\hline 4 & $\begin{array}{l}\text { Ulimoen } \\
\text { et al. }\end{array}$ & $\begin{array}{l}\text { Cross } \\
\text { sectional } \\
\text { cohort } \\
\text { study }\end{array}$ & $\begin{array}{l}48 \text { T2DM patients } \\
\text { aging between } \\
18 \text { to } 75 \text { years } \\
\text { without CAD } \\
\text { symptoms }\end{array}$ & CCTA ICA & $\begin{array}{c}\text { Detection of } \\
\text { significant CAD }\end{array}$ & $\begin{array}{c}\text { For the exclusion of significant } \\
\text { CAD in T2DM patients, } \\
\text { CCTA in comparison with } \\
\text { ICA offers equal sensitivity } \\
(100 \%) \text { and negative } \\
\text { predictive value }(100 \%) .\end{array}$ \\
\hline 5 & $\begin{array}{l}\text { Shimabukuro } \\
\text { et al. }\end{array}$ & $\begin{array}{l}\text { Prospective } \\
\text { cohort } \\
\text { study }\end{array}$ & $\begin{array}{l}506 \text { T2DM patients } \\
\text { asymptomatic } \\
\text { for CAD }\end{array}$ & $\operatorname{MDCT}(\mathrm{CCTA})$ & $\begin{array}{c}\text { Prevalence and } \\
\text { severity of stenosis, } \\
\text { atherosclerosis } \\
\text { Sex-related risk } \\
\text { factors suggestive of } \\
\text { asymptomatic CAD }\end{array}$ & $\begin{array}{c}82 \% \text { and } 72 \% \text { of patients } \\
\text { (men and women) with DM } \\
\text { with no CV symptoms had } \\
\text { abnormal findings in CCTA. } \\
\text { Factors predicting CAD in men } \\
\text { were HbA1c } \geq 7.4 \%(p=0.003), \\
\text { dyslipidemia ( } p=0.004), \\
\text { duration of diabetes } \\
(p=0.004), \text { retinopathy } \\
(p=0.004), \text { and other type of } \\
\text { cardiovascular ( } p=0.021) \\
\text { and in women were } \\
\text { duration of diabetes }(p=0.041) \\
\text { and retinopathy }(p=0.000) .\end{array}$ \\
\hline 6 & $\begin{array}{l}\text { Zafrir } \\
\text { et al. }\end{array}$ & $\begin{array}{l}\text { Cohort } \\
\text { prospective } \\
\text { study }\end{array}$ & $\begin{array}{c}600 \text { patients } 55 \\
\text { to } 74 \text { years old with } \\
\text { asymptomatic T2DM }\end{array}$ & $\begin{array}{c}\text { Measuring } \\
\text { cardiorespiratory } \\
\text { fitness through } \\
\text { metabolic equivalents } \\
\text { METS during exercise, } \\
\text { Non-enhanced CT }\end{array}$ & $\begin{array}{l}\text { All-cause } \\
\text { mortality, MI } \\
\text { and stroke }\end{array}$ & $\begin{array}{l}\text { Low CRF can increase the } \\
\text { risk of all-cause mortality } \\
\text { in DM despite low CAC score. } \\
(\mathrm{HR}=2.36,95 \% \mathrm{CI}= \\
1.49-3.75, p<0.00001) .\end{array}$ \\
\hline 7 & $\begin{array}{l}\text { Halon } \\
\text { et al. }\end{array}$ & $\begin{array}{l}\text { Prospective } \\
\text { cohort study }\end{array}$ & $\begin{array}{l}630 \text { T2DM patients } \\
\text { between } 55 \text { to } \\
74 \text { years old, } \\
\text { without known CAD }\end{array}$ & CTA & $\begin{array}{c}\text { Cardiovascular } \\
\text { death, non-fatal MI, } \\
\text { unstable angina or } \\
\text { new onset angina } \\
\text { were considered as } \\
\text { primary and non- } \\
\text { coronary vascular } \\
\text { events as secondary } \\
\text { outcomes }\end{array}$ & $\begin{array}{l}\text { The UKPDS and the Gensini } \\
\text { score were independent } \\
\text { predictive factors for primary } \\
\text { CHD events respectively } \\
{[(\mathrm{HR}=1.3,95 \% \mathrm{CI}=1.1-1.5,} \\
p=0.003) \text { and }(\mathrm{HR}=3.2, \\
95 \% \mathrm{CI}=2.1-5.0, p<0.0001)] \text {. } \\
\text { The use of total plaque burden, } \\
\text { Gensini, UKPDS and CAC. }\end{array}$ \\
\hline
\end{tabular}


Table II. Continued

\begin{tabular}{|c|c|c|c|c|c|c|}
\hline Entry & Study & Design & Population & $\begin{array}{l}\text { Method of } \\
\text { screening }\end{array}$ & $\begin{array}{l}\text { Clinical } \\
\text { outcome }\end{array}$ & Conclusion \\
\hline & & & & & & $\begin{array}{l}\text { score all together has the } \\
\text { greatest predictive value in } \\
\text { discriminating primary CHD } \\
\text { events }(\mathrm{C} 0.824,95 \% \mathrm{CI}= \\
0.768-0.881, p=0.021)\end{array}$ \\
\hline 8 & $\begin{array}{l}\text { Srinivasan } \\
\quad \text { et al. }\end{array}$ & $\begin{array}{c}\text { Cross } \\
\text { sectional } \\
\text { study }\end{array}$ & $\begin{array}{c}175 \text { individuals } \\
\text { between } 45-65 \\
\text { years old, divided } \\
\text { in } 4 \text { groups, non-DM, } \\
\text { DM less than } 5, \text { between } \\
5-10 \text { years or more } \\
\text { than } 10 \text { years }\end{array}$ & $\begin{array}{l}\text { Coronary } \\
\text { angiogram }\end{array}$ & $\begin{array}{l}\text { Coronary profile } \\
\text { of non-DM, and } \\
\text { T2DM patients } \\
\text { according to the } \\
\text { diabetic duration. }\end{array}$ & $\begin{array}{l}\text { Patients with DM more than } \\
5 \text { years had higher scores in } \\
\text { angiogram than those with } \\
\text { less than } 5 \text { years }(p<0.05) \text { and } \\
\text { show greater structural changes } \\
\text { in coronary arteries. No such } \\
\text { significant differences appeared } \\
\text { between 5-10 years and more } \\
\text { than } 10 \text { years with } \\
\text { T2DM }(p>0.05) .\end{array}$ \\
\hline 9 & $\begin{array}{l}\text { Christensen } \\
\quad \text { et al. }\end{array}$ & $\begin{array}{l}\text { Prospective } \\
\text { cohort } \\
\text { study }\end{array}$ & $\begin{array}{l}200 \mathrm{~T} 2 \mathrm{DM} \text { patients } \\
\text { without known CAD } \\
\text { and elevated excretion } \\
\text { rate of urinary albumin }\end{array}$ & $\begin{array}{l}\text { U/S, Cardiac } \\
\text { CT, CIMT }\end{array}$ & $\begin{array}{c}\text { Cardiovascular events } \\
\text { and mortality }\end{array}$ & $\begin{array}{l}\text { Patients with DM and elevated } \\
\text { CAT had higher risk of CVD } \\
\text { and mortality than patients with } \\
\text { low CAT }(\mathrm{HR}=2.0,95 \% \mathrm{CI}= \\
1.1-3.7, p=0.017) \text {. Total cardiac } \\
\text { adipose tissue is correlated } \\
\text { with IL-8 }(p<0.039) .\end{array}$ \\
\hline 10 & $\begin{array}{l}\text { Nezarat } \\
\text { et al. }\end{array}$ & $\begin{array}{l}\text { Case control } \\
\text { prospective } \\
\text { study }\end{array}$ & $\begin{array}{l}181 \text { subjects aging } \\
\text { between } 25 \text { and } 40 \text { years } \\
\text { old divided into T2DM } \\
\text { and non-DM group }\end{array}$ & $\begin{array}{c}\text { CAC scanning, } \\
\text { CCTA }\end{array}$ & $\begin{array}{c}\text { Prevalence and severity } \\
\text { of early coronary } \\
\text { atherosclerotic } \\
\text { disease }\end{array}$ & $\begin{array}{c}\text { Young patients with DM } \\
\text { had a prevalence of } \\
\text { subclinical CAD of } 58 \% \\
\text { compared with } 20 \% \text { in } \\
\text { non-DM }(p<0.001) . \\
\text { Young DM had } 25 \% \text { risk } \\
\text { of getting CAC VS non-DM } \\
\text { (RR }=1.253,95 \% \text { CI }= \\
1.049-1.497, p=0.013) .\end{array}$ \\
\hline 11 & $\begin{array}{l}\text { Shalaeva } \\
\text { et al. }\end{array}$ & $\begin{array}{l}\text { Prospective } \\
\text { cohort } \\
\text { study }\end{array}$ & $\begin{array}{l}179 \text { patients with } \\
\text { T2DM, known CAD } \\
\text { and trans-femoral } \\
\text { amputation }\end{array}$ & CCTA & $\begin{array}{l}\text { Major Adverse } \\
\text { Cardiovascular } \\
\text { Event (MACE) } \\
\text { at } 1 \text { year }\end{array}$ & $\begin{array}{c}\text { Patients with greater CCTA } \\
\text { stenonis including more } \\
\text { vessels had lower MACE - } \\
\text { free survival rate }(p<0.0001) \\
\text { Compliance of patients } \\
\text { decreases risk of MACE. }\end{array}$ \\
\hline 12 & $\begin{array}{l}\mathrm{Wu} \\
\text { et al. }\end{array}$ & $\begin{array}{l}\text { Prospective } \\
\text { cohort } \\
\text { study }\end{array}$ & $\begin{array}{l}1584 \text { patients with } \\
\text { T2DM aging } \\
20 \text { years and above }\end{array}$ & B-mode $\mathrm{U} / \mathrm{S}$ & $\begin{array}{l}\text { Atherosclerosis, } \\
\text { evaluation of CHD } \\
\text { and stroke events }\end{array}$ & $\begin{array}{c}\text { Carotid atherosclerosis } \\
\text { is an independent risk } \\
\text { factor for } \mathrm{CHD}(\mathrm{OR}=2.66, \\
95 \% \mathrm{CI}=2.05-3.46 p=0.00) \\
\text { and for strokes }(\mathrm{OR}=3.11, \\
95 \% \mathrm{CI}=2.38-4.07, p<0.001) .\end{array}$ \\
\hline 13 & $\begin{array}{l}\text { Halon } \\
\text { et al. }\end{array}$ & $\begin{array}{l}\text { Prospective } \\
\text { cohort } \\
\text { study }\end{array}$ & $\begin{array}{c}735 \text { patients } 55 \text { to } \\
74 \text { years old with T2DM } \\
\text { without known CAD or HF }\end{array}$ & CTA & $\begin{array}{l}\text { Death (primary) and } \\
\text { cardiovascular events } \\
\text { caused by HF }\end{array}$ & $\begin{array}{c}\text { Independent predictors } \\
\text { of HF-CVD events in low to } \\
\text { intermediate risk } \\
\text { asymptomatic T2DM were: } \\
\text { LA/RA ratio }>1(\mathrm{HR}=4.8, \\
95 \% \mathrm{CI}=2.2-10.4, p<0.0001) \\
>\text { Microvascular disease }(\mathrm{HR}=5.1, \\
95 \% \mathrm{CI}=2.5-10.7, p<0.0001) \\
\text { Systolic } \mathrm{BP}(\mathrm{HR}=1.2, \\
95 \% \mathrm{CI}=1.0-1.4, \mathrm{p}=0.035)\end{array}$ \\
\hline
\end{tabular}

CAD: Coronary artery disease; LDL: low density lipoprotein; SdLDL: small dense low density lipoprotein; T2DM: Type 2 diabetes mellitus; MACE: major adverse cardiovascular event; MI: myocardial infraction; HF: heart failure; CHD: coronary heart disease; ACS: acute coronary syndrome; CCTA: coronary computed tomography angiography; MDCT: multi-detector computed tomography; CAC: coronary artery calcium; ABI: ankle brachial index; CAVI: cardio-ankle vascular index; ICA: invasive coronary angiography; CRF: cardio-respiratory fitness; METs: metabolic equivalents; U/S: ultra-sound; CIMT: carotid intima-media thickness; LA/RA: left atrium/right atrium. 


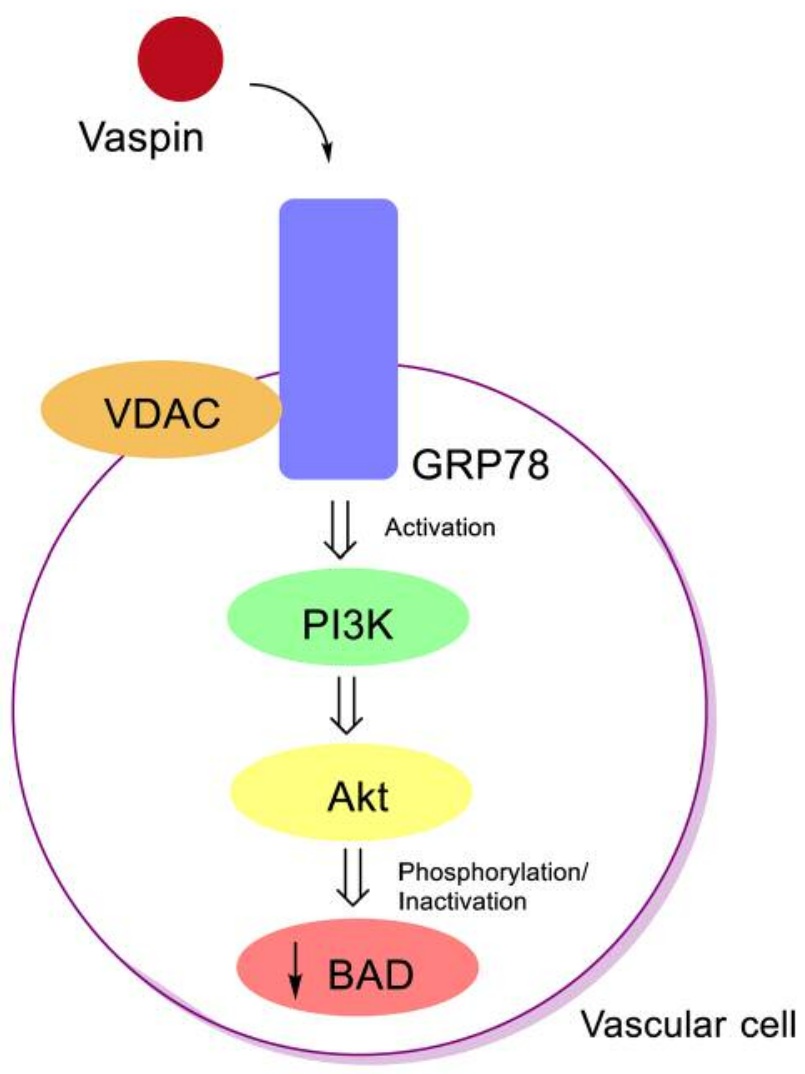

Figure 2. Anti-apoptotic pathway of vaspin in vascular cells.

retinopathy and longer duration of diabetes were more susceptible to CAD. As a result, the combination of MDCT with the assessment of risk factors, described above, constitutes a potential screening tool for asymptomatic CAD in type 2 diabetes (39).

Moreover, in another study, cardiorespiratory fitness (CRF) [in peak metabolic equivalents (METs)] and CAC scores of a group of 600 asymptomatic type 2 diabetic individuals were measured via computed tomography (Table II) (40). According to the results, low CRF offers a positive prognostic value for all-cause mortality, stroke and myocardial infraction in asymptomatic diabetics, despite a low CAC. Therefore, lifestyle changes and assessment of other risk factors for cardiovascular problems could be beneficial to patients with low CRF. The study also demonstrated the additive value of CRF along with CAC for the identification of high risk asymptomatic diabetic patients (40).

One year later, a study of 630 diabetic subjects without known CAD underlined that a combination of CCTA with clinical risk stratification methods and coronary artery calcium score is effective in detecting patients with greater risk of primary cardiovascular events (Table II) (41).
In 2016, Srinivasan et al., tested 175 individuals who underwent coronary angiogram for the detection of CAD (Table II). The outcome of the research indicated that there is a correlation between the duration of diabetes and the existence of CAD. Remarkably, patients with more than 5 years with diabetes showed greater vascular structural changes than patients with less than 5 years with diabetes. Therefore, it is of paramount importance to intervene diagnostically and therapeutically in the first 5 years of type 2 diabetes (42).

Next year, Christensen et al., examined a group of 200 patients who underwent echocardiography for the screening of cardiac adipose tissue (CAT) (Table II). According to the results, higher than normal levels of CAT are correlated with greater risk of mortality and cardiovascular incident. In addition, CAT is linked to inflammation, as it is positively associated with IL-8 (43).

In 2017, Nezarat et al., investigated the prevalence and severity of early coronary atherosclerotic disease in 181 patients less than 40 years old (Table II). According to the clinical outcome, type 2 diabetic patients are prone to developing CAD more often and with greater extent, depending on the type of atheromatic plaques, compared to non-diabetic individuals of matched-age. As a result, computed tomography angiography may be used as a detector of subclinical atherosclerosis in this group (44).

In addition, a group of scientists have evaluated the effect of patient's compliance on the prevention of major adverse cardiovascular event (MACE) in 179 type 2 diabetic patients who underwent coronary angiography before trans-femoral amputation for 1 year (Table II) (45). They proved that noncompliant patients presented more MACEs in comparison with the compliant ones. Additionally, the non-compliant patients with more severe CAD (defined by CCTA), depending on the extent and the position of the lesion, revealed a worse clinical outcome than the non-compliant patients with less severe lesion (45).

In 2017, $\mathrm{Wu}$ et al., tested 1,584 individuals who underwent ultrasound screening for carotid atherosclerosis (CA) (Table II) (46). They demonstrated that the prevalence of carotid atherosclerosis is greater in type 2 diabetics than in non-diabetic patients. Moreover, type 2 diabetic men and elderlies were susceptible to develop CA (gender and agerelated). Last but not least, CA was positively related to the appearance of stroke and coronary heart events, setting CA monitoring a necessity (46).

The same year, another study investigated whether cardiovascular death and heart failure are affected by CCTA findings and clinical factors in 735 type 2 diabetic subjects who underwent CCTA and clinical assessment (Table II) (47). The outcome of the research implies that left/right atrial (LA/RA) volume ratio $>1$ (defined by CCTA), the existence of microvascular disease (retinopathy and nephropathy) and 
increased systolic blood pressure lead to heart failure and cardiovascular death in asymptomatic patients of low to intermediate risk. Thus, taking these factors under consideration will possibly ameliorate the clinical result (47).

\section{Conclusion}

As we have seen in this review, researchers used imaging methods, blood markers and clinical exercises to identify screening tools for CAD. A broad range of factors were investigated, utilizing large groups of subjects suffering from type 2 diabetes. Significant results were obtained from all the studies, described in this review. As CAD reflects one of the most important complications of type 2 diabetes mellitus, it is of paramount importance to find new and more efficient methods to predict CAD both in time and extent and offer clinical benefits to patients. Further research should be performed in order to identify factors predicting high risk diabetic patients for coronary events.

\section{Conflicts of Interest}

All Authors declare that there are no conflicts of interest.

\section{Authors' Contributions}

AP and ED designed the study. AP, PF and ED wrote the article. AG, CD, and NG collected the data. AG and DM revised the article.

\section{References}

1 International Diabetes Federation. IDF Diabetes Atlas. 6th ed. IDF, Brussels: 2013.

2 Bos $\mathrm{M}$ and Agyemang C: Prevalence and complications of diabetes mellitus in Northern Africa, a systematic review. BMC Public Health 13: 387, 2013. PMID: 23617762. DOI: 10.1186/ 1471-2458-13-387

3 Albers AR, Krichavsky MZ and Balady GJ: Stress testing in patients with diabetes mellitus. Circulation 113: 583-592, 2006. PMID: 16449735 . DOI: 10.1161/CIRCULATIONAHA.105.584524

4 Lusis AJ: Atherosclerosis. Nature 407: 233-241, 2000. PMID: 11001066. DOI: $10.1038 / 35025203$

5 Ross R: Atherosclerosis is an inflammatory disease. Am Heart J 138(5 Pt 2): 419-420, 1999. PMID: 10539839.

6 Hansson GK, Robertson AK and Söderberg-Nauclér C: Inflammation and atherosclerosis. Annu Rev Pathol 1: 297-329, 2006. PMID: 18039117. DOI: 10.1146/annurev.pathol.1.110304 100100

7 Gawaz M, Langer H and May AE: Platelets in inflammation and atherogenesis. J Clin Invest 115: 3378-3384, 2005. PMID: 16322783. DOI: $10.1172 /$ JCI27196

8 May AE, Kälsch T, Massberg S, Herouy Y, Schmidt R and Gawaz M: Engagement of glycoprotein IIb/IIIa $(\alpha(\mathrm{IIb}) \beta 3)$ on platelets upregulates CD40L and triggers CD40L-dependent matrix degradation by endothelial cells. Circulation 106: 2111 2117, 2002. PMID: 12379582.
9 Libby P, Ridker PM and Hansson GK: Progress and challenges in translating the biology of atherosclerosis. Nature 473: $317-$ 325, 2011. PMID: 21593864. DOI: 10.1038/nature10146

10 Weber C, Zernecke A and Libby P: The multifaceted contributions of leukocyte subsets to atherosclerosis: lessons from mouse models. Nat Rev Immunol 8: 802-815, 2008. PMID: 18825131. DOI: $10.1038 /$ nri2415

11 Sanchis-Gomar F, Perez-Quilis C, Leischik R and Lucia A: Epidemiology of coronary heart disease and acute coronary syndrome. Ann Transl Med 4: 256, 2016. PMID: 27500157. DOI: $10.21037 / \mathrm{atm} .2016 .06 .33$

12 Libby P and Theroux P: Pathophysiology of coronary artery disease. Circulation 111: 3481-488, 2005. PMID: 15983262. DOI: $10.1161 /$ CIRCULATIONAHA.105.537878

13 Haffner SM, Lehto S, Ronnemaa T, Pyorala K and Laakso M: Mortality from coronary heart disease in subjects with type 2 diabetes and in nondiabetic subjects with and without prior myocardial infarction. N Engl J Med 339: 229-234, 1998. PMID: 9673301. DOI: 10.1056/NEJM199807233390404

14 Bonora E, Formentini G, Calcaterra F, Lombardi S, Marini F, Zenari L, Saggiani F, Poli M, Perbellini S, Raffaelli A, Cacciatori V, Santi L, Targher G, Bonadonna R and Muggeo M: HOMA-estimated insulin resistance is an independent predictor of cardiovascular disease in type 2 diabetic subjects: prospective data from the Verona Diabetes Complications Study. Diabetes Care 25: 1135-1141, 2002. PMID: 12087010.

15 Huang YC, Chang PY, Hwang JS and Ning HC: Association of small dense low density lipoprotein cholesterol in type 2 diabetics with coronary artery disease. Biomed J 37: 375-379, 2014. PMID: 25179702. DOI: 10.4103/2319-4170.132883

16 Dev K, Sharma SB, Garg S, Aggarwal A and Madhu SV: Glycated apolipoprotein B-A surrogate marker of subclinical atherosclerosis. Diabetes Metab Syndr 10: 78-81, 2016. PMID: 26614298. DOI: 10.1016/j.dsx.2015.09.012

17 Chen J, Zhang Y, Liu J, Chen MH, Guo YL, Zhu CG, Xu RX, Dong Q and Li JJ: Role of lipoprotein(a) in predicting the severity of new on-set coronary artery disease in type 2 diabetics: A Gensini score evaluation. Diabetes Vasc Dis Res 12: 258-264, 2015. PMID: 25861813. DOI: 10.1177/147916411 5579004

18 Gouni-Berthold I and Berthold HK: Lipoprotein(a): current perspectives. Curr Vasc Pharmacol 9: 682-692, 2011. PMID: 21529331.

19 Ozturk D, Celik O, Satilmis S, Aslan S, Erturk M, Cakmak HA, Kalkan AK, Ozyilmaz S, Diker V and Gul M: Association between serum galectin-3 levels and coronary atherosclerosis and plaque burden/structure in patients with type 2 diabetes mellitus. Coron Artery Dis 26: 396-401, 2015. PMID: 25887000. DOI: $10.1097 / \mathrm{MCA} .0000000000000252$

20 Rubinstein N, Ilarregui JM, Toscano MA and Rabinovich GA: The role of galectins in the initiation, amplification and resolution of the inflammatory response. Tissue Antigens 64: 112, 2004. PMID: 15191517. DOI: 10.1111/j.0001-2815.2004.002 78.x

21 Srinivasan MP, Kamath PK, Bhat NM, Pai ND, Manjrekar PA and Mahabala C: Factors associated with no apparent coronary artery disease in patients with type 2 diabetes mellitus for more than 10 years of duration: A case control study. Cardiovasc Diabetol 14: 146-152, 2015. PMID: 26521236. DOI: 10.1186/ s12933-015-0307-z 
22 Tasevska I, Enhörning S, Persson M, Nilsson PM and Melander $\mathrm{O}$ : Copeptin predicts coronary artery disease cardiovascular and total mortality. Heart 102: 127-132, 2016. PMID: 26661323. DOI: 10.1136/heartjnl-2015-308183

23 Enhorning S, Wang TJ, Nilsson PM, Almgren P, Hedblad B, Berglund G, Struck J, Morgenthaler NG, Bergmann A, Lindholm E, Groop L, Lyssenko V, Orho-Melander M, Newton-Cheh C and Melander O: Plasma copeptin and the risk of diabetes mellitus. Circulation 121: 2102-2108, 2010. PMID: 20439785. DOI: $10.1161 /$ CIRCULATIONAHA.109.909663

24 Morgenthaler NG, Struck J, Alonso C and Bergmann A: Assay for the measurement of copeptin, a stable peptide derived from the precursor of vasopressin. Clin Chem 52: 112-119, 2006. PMID: 16269513. DOI: 10.1373/clinchem.2005.060038

25 Abbasi A, Corpeleijn E, Meijer E, Postmus D, Gansevoort RT, Gans RO, Struck J, Hillege HL, Stolk RP, Navis G and Bakker SJ: Sex differences in the association between plasma copeptin and incident type 2 diabetes: The Prevention of Renal and Vascular Endstage Disease (PREVEND) study. Diabetologia 55: 1963-1970, 2012. PMID: 22526609. DOI: 10.1007/s00125-0122545-x

26 Paul Chubb SA, Davis WA, Peters KE and Davis TM: Serum bicarbonate concentration and the risk of cardiovascular disease and death in type 2 diabetes: the Fremantle Diabetes Study. Cardiovasc Diabetol 15: 143, 2016. PMID: 27716263. DOI: 10.1186/s12933-016-0462-x

27 Hao F, Zhang HJ, Zhu J, Kuang H, Yu Q, Bai M and Mu J: Association between vaspin level and coronary artery disease in patients with type 2 diabetes. Diabetes Res Clin Pract 113: 2632, 2016. PMID: 26972957. DOI: 10.1016/j.diabres.2015.12.001

28 Aust G, Richter O, Rohm S, Kerner C, Hauss J, Klöting N, Ruschke K, Kovacs P, Youn BS and Blüher M: Vaspin serum concentrations in patients with carotid stenosis. Atherosclerosis 204: 262-266, 2009. PMID: 18848328. DOI: 10.1016/j.athero sclerosis.2008.08.028

29 Jung CH, Lee WJ, Hwang JY, Seol SM, Kim YM, Lee YL and Park JY: Vaspin protects vascular endothelial cells against free fatty acid-induced apoptosis through a phosphatidylinositol 3kinase/Akt pathway. Biochem Biophys Res Commun 413: 264 269, 2011. PMID: 21893030. DOI: 10.1016/j.bbrc.2011.08.083

30 Nakatsuka A, Wada J, Iseda I, Teshigawara S, Higashio K, Murakami K, Kanzaki M, Inoue K, Terami T, Katayama A, Hida K, Eguchi J, Ogawa D, Matsuki Y, Hiramatsu R, Yagita H, Kakuta S, Iwakura Y and Makino H: Vaspin inhibits apoptosis of endothelial cells as a ligand for cell-surface GRP78/VDAC complex. Circ Res 112: 771-780, 2013. PMID: 23307819. DOI: 10.1161/CIRCRESAHA.111.300049

31 Müller KA, Rath D, Schmid M, Schoenleber H, Gawaz M, Geisler T and Müller II: High plasma levels of gremlin-1 and macrophage migration inhibitory factor, but not their ratio, indicate an increased risk for acute coronary syndrome in patients with type 2 diabetes mellitus. Clin Cardiol 39: 201-206, 2016. PMID: 27101443. DOI: 10.1002/clc.22509

32 Müller II, Müller KA, Karathanos A, Schönleber H, Rath D, Vogel S, Chatterjee M, Schmid M, Haas M, Seizer P, Langer H, Schaeffeler E, Schwab M, Gawaz M and Geisler T: Impact of counterbalance between macrophage migration inhibitory factor and its inhibitor Gremlin-1 in patients with coronary artery disease. Atherosclerosis 237: 426-432, 2014. PMID: 25463068. DOI: 10.1016/j.atherosclerosis.2014.09.010
33 Müller II, Schonberger T, Schneider M, Borst O, Ziegler M, Seizer P, Leder C, Müller K, Lang M, Appenzeller F, Lunov O, Büchele B, Fahrleitner M, Olbrich M, Langer H, Geisler T, Lang F, Chatterjee M, de Boer JF, Tietge UJ, Bernhagen J, Simmet T and Gawaz M: Gremlin-1 is an inhibitor of macrophage migration inhibitory factor and attenuates atherosclerotic plaque growth in $\mathrm{ApoE}^{-/-}$Mice. J Biol Chem 288: 31635-31645, 2013. PMID: 24003215. DOI: 10.1074/jbc.M113.477745

34 Valensi P, Avignon A, Sultan A, Chanu B, Nguyen MT and Cosson E: Atherogenic dyslipidemia and risk of silent coronary artery disease in asymptomatic patients with type 2 diabetes: a cross sectional study. Cardiovasc Diabetol 15: 104-113, 2016. PMID: 27450534. DOI: 1186/s12933-016-0415-4

35 Park GM, Lee SW, Cho YR, Kim CJ, Cho JS, Park MW, Her SH, Ahn JM, Lee JY, Park DW, Kang SJ, Kim YH, Lee CW, Koh EH, Lee WJ, Kim MS, Lee KU, Kang JW, Lim TH, Park SW, Park SJ and Park JY: Coronary computed tomographic angiographic findings in asymptomatic patients with type 2 diabetes mellitus. Am J Cardiol 113: 765-771, 2014. PMID: 24528613. DOI: 10.1016/j.amjcard.2013.11.028

36 Park GM, Lee JH, Lee SW, Yun SC, Kim YH, Cho YR, Gil EH, Kim TS, Kim CJ, Cho JS, Park MW, Her SH, Yang DH, Kang JW, Lim TH, Koh EH, Lee WJ, Kim MS, Lee KU, Kim HK, Choe J and Park JY: Comparison of coronary computed tomographic angiographic findings in asymptomatic subjects with versus without diabetes mellitus. Am J Cardiol 116: 372-378, 2015. PMID: 26037293. DOI: 10.1016/j.amjcard. 2015.04.046

37 Chung SL, Yang CC, Chen CC, Hsu YC and Lei MH: Coronary artery calcium score compared with cardio-ankle vascular index in the prediction of cardiovascular events in asymptomatic patients with type 2 diabetes. J Atheroscler Thromb 22: 12551265, 2015. PMID: 26269147. DOI: 10.5551/jat.29926

38 Ulimoen GR, Ofstad AP, Endresen K, Gullestad L, Johansen OE and Borthne A: Low-dose CT coronary angiography for assessment of coronary artery disease in patients with type 2 diabetes - a cross-sectional study. BMC Cardiovasc Disord 15: 147-153, 2015. PMID: 26573616. DOI: 10.1186/s12872-0150143-9

39 Shimabukuro M, Saito T, Higa T, Nakamura K, Masuzaki H and Sata M; Fukuoka diabetologists group: Risk stratification of coronary artery disease in asymptomatic diabetic subjects using multidetector computed tomography. Circ J 79: 2422-2429, 2015. PMID: 26399764. DOI: 10.1253/circj.CJ-15-0325

40 Zafrir B, Azaiza M, Gaspar T, Mery ID, Azencot M, Lewis BS, Rubinshtein R and Halon DA: Low cardiorespiratory fitness and coronary artery calcification: Complementary cardiovascular risk predictors in asymptomatic type 2 diabetics. Atherosclerosis 241: 634-640, 2015. PMID: 26117400. DOI: 10.1016/j.athero sclerosis.2015.06.020

41 Halon DA, Azencot M, Rubinshtein R, Zafrir B, Flugelman MY and Lewis BS: Coronary computed tomography (CT) angiography as a predictor of cardiac and noncardiac vascular events in asymptomatic type 2 diabetics: A 7-year populationbased cohort study. J Am Heart Assoc 5: e003226, 2016. PMID: 27412899. DOI: $10.1161 /$ JAHA.116.003226

42 Srinivasan MP, Kamath PK, Bhat NM, Pai ND, Bhat RU, Shah TD, Singhal A and Mahabala C: Severity of coronary artery disease in type 2 diabetes mellitus: Does the timing matter? Indian Heart J 68: 158-163, 2016. PMID: 27133324. DOI: 10.1016/j.ihj.2015.08.004 
43 Christensen RH, Scholten BJV, Hansen CS, Heywood SE Rosenmeier JB, Andersen UB, Hovind P, Reinhard H, Parving $\mathrm{HH}$, Pedersen BK, Jørgensen ME, Jacobsen PK and Rossing P: Epicardial, pericardial and total cardiac fat and cardiovascular disease in type 2 diabetic patients with elevated urinary albumin excretion rate. Eur J Prev Cardiol 24: 1517-1524, 2017. PMID: 28650207. DOI: $10.1177 / 2047487317717820$

44 Nezarat N, Budoff MJ, Luo Y, Darabian S, Nakanishi R, Li D, MD, Sheidaee N, Kim M, BSa, Alani A, Matsumoto S, Rahmani S, Kanisawa M, Ceponiene I, Osawa K, Qi H, Hamal S, Kitslaar P, Broersen A, Flores F, Ipp E and Khazai B: Presence, characteristics, and volumes of coronary plaque determined by computed tomography angiography in young type 2 diabetes mellitus. Am J Cardiol 119: 1566-1571, 2017. PMID: 28343599. DOI: $10.1016 /$ j.amjcard.2017.02.023

45 Shalaeva EV, Saner H, Janabaev BB and Shalaeva AV: Tenfold risk increase of major cardiovascular events after high limb amputation with non-compliance for secondary prevention measures. Eur J Prev Cardiol 24: 708-716, 2017. PMID: 28071959. DOI: $10.1177 / 2047487316687103$
46 Wu Y, He J, Sun X, Zhao YM, Lou HY, Ji XL, Pang XH, Shan LZ, Kang YX, Xu J, Zhang SZ, Wang YJ, Ren YZ and Shan PF: Carotid atherosclerosis and its relationship to coronary heart disease and stroke risk in patients with type 2 diabetes mellitus. Medicine (Baltimore) 96: e8151, 2017. PMID: 28953658. DOI: 10.1097/MD.0000000000008151

47 Halon DA, Ayman J, Rubinshtein R, Zafrir B, Azencot M and Lewis BS: Cardiac computed tomography angiographic findings as predictors of late heart failure in an asymptomatic diabetic cohort: An 8-year prospective follow-up study. Cardiology 138: 218-227, 2017. PMID: 28817814. DOI: 10.1159/000478995

Received March 16, 2019

Revised April 28, 2019

Accepted April 30, 2019 